\title{
Adjunctive treatment with eplerenone reduced morbidity and mortality in acute myocardial infarction
}

Pitt B, Remme W,Zannad F, et al. Eplerenone, a selective aldosterone blocker, in patients with left ventricular dysfunction after myocardial infarction. N Engl J Med 2003;348:1309-21.

QUESTION: In patients with acute myocardial infarction (MI) complicated by left ventricular dysfunction and congestive heart failure (CHF), does adjunctive treatment with eplerenone reduce morbidity and mortality more than placebo?

Design

Randomised \{allocation concealed $*\}$, blinded (clinicians, patients, outcome assessors, \{data collectors, data analysts, and manuscript writers $\} \uparrow$ ), ${ }^{*}$ placebo controlled trial with mean 16 months of follow up (Eplerenone Post-Acute Myocardial Infarction Heart Failure Efficacy and Survival Study [EPHESUS]).

\section{Setting}

674 centres in 37 countries.

\section{Patients}

6642 patients (mean age $64 \mathrm{y}, 71 \%$ men) with acute MI, left ventricular dysfunction (ejection fraction $\leq 40 \%$ ), and HF (confirmed by the presence of pulmonary rales, pulmonary venous congestion on chest radiography, or a third heart sound). Exclusion criteria included potassium sparing diuretics, serum creatinine $\geq 220$ $\mu \mathrm{mol} / \mathrm{l}$, and serum potassium $>5.0 \mathrm{mmol} / \mathrm{l}$ before randomisation. 6632 patients $(99.8 \%)$ were included in the follow up analysis.

\section{Intervention}

Patients were stratified by clinical site and allocated 3-14 days after acute MI to eplerenone, $25 \mathrm{mg}$ /day (increased to a maximum of $50 \mathrm{mg} / \mathrm{d}$ after $4 \mathrm{wk})(\mathrm{n}=3319)$ or placebo (n=3313). All patients received optimal medical treatment (angiotensin converting enzyme inhibitors, angiotensin receptor blockers, diuretics, $\beta$ blockers, and coronary reperfusion treatment).

\section{Main outcome measures}

Time to death from any cause and time to death from cardiovascular $(\mathrm{CV})$ causes or first hospital admission for a CV event (heart failure, recurrent acute MI, stroke, or ventricular arrhythmia). Secondary outcomes were death from $\mathrm{CV}$ causes, death from any cause, or any hospital admission.

\section{Main results}

Analysis was by intention to treat. Fewer patients in the eplerenone group died from any cause, died from CV causes, or were admitted to hospital for CV events than did those in the placebo group (table). Secondary endpoints were also reduced in eplerenone recipients (table). More patients in the eplerenone group had serious hyperkalaemia (serum potassium concentration

Source of funding: Pharmacia.

For correspondence: Dr B Pitt, University of Michigan, Ann Arbor, MI, USA.

bpitt@umich.edu

Abstract and commentary also appear in ACP Journal Club. $\geq 6.0 \mathrm{mmol} / \mathrm{l}$ ) than did patients in the placebo group $(5.5 \%$ v $3.9 \%, \mathrm{p}=0.002)$.

\section{COMMENTARY}

The results of the EPHESUS study by Pitt $e t$ al and the previously published Randomised Aldactone Evaluation Study (RALES) ${ }^{1}$ provide strong evidence for the addition of an aldosterone antagonist to optimal conventional treatment in patients with $\mathrm{CHF}$ and reduced left ventricular systolic function.

EPHESUS establishes the role of selective aldosterone antagonism with eplerenone in patients with an $\mathrm{EF} \leq 40 \%$ and clinical signs of CHF within 3-14 days of an acute MI. Debate will probably focus on whether these results are specific to selective aldosterone antagonists (eplerenone) or whether non-selective agents (spironolactone) could provide similar results (particularly if there is a marked price difference).

Are there strong reasons to believe that the potential mechanisms of benefit are unique to eplerenone rather than spironolactone in the postinfarction subgroup of patients with CHF? Probably not. In addition, the magnitude of benefit with spironolactone in the RALES study (in which 54\% of patients had an ischaemic basis for CHF) was twice as great in relative terms as, and 4 times greater in absolute terms than, eplerenone in the EPHESUS study. Potential reasons for these differences include the sicker population studied, early termination of the trial, and the scarce use of $\beta$ blockers (which were not yet established as CHF treatment) in the RALES study. A head to head comparison of the 2 drugs would determine whether true differences exist between them.

Although both drugs were well tolerated, a major difference was that men receiving spironolactone had a $10 \%$ risk of gynaecomastia or breast pain, which was not seen in men receiving eplerenone.

Overall, the results of the EPHESUS and RALES studies are impressive and warrant the early addition of an aldosterone antagonist (whether eplerenone or spironolactone) for preventing or delaying the considerable mortality and morbidity associated with clinical left ventricular dysfunction caused by MI. These trials also show the need for closer attention to the possibility of hyperkalaemia, particularly in patients with impaired renal function.

Manu Prabhakar, MD David Massel, MD

London Health Sciences Centre, London, Ontario, Canada

1 Pitt B, Zannad F, Remme WJ, et al. N Engl J Med 1999;341: 709-17.

\section{Conclusion}

In patients with acute myocardial infarction complicated by left ventricular dysfunction and heart failure, adjunctive treatment with eplerenone reduced morbidity and mortality more than placebo.

*See glossary.

†Information provided by author.

Eplerenone v placebo for myocardial infarction at mean 16 months

\begin{tabular}{lllll}
\hline Outcomes & Eplerenone & Placebo & RRR (95\% CI) & NNT (CI) \\
Death from any cause & $14 \%$ & $17 \%$ & $15 \%(4$ to 25$)$ & $44(25$ to 174$)$ \\
\hline Death from CV causes or CV event hospital admission & $27 \%$ & $30 \%$ & $13 \%(5$ to 21$)$ & $31(19$ to 88$)$ \\
\hline Death from any cause or any hospital admission & $52 \%$ & $55 \%$ & $8 \%(2$ to 14$)$ & $33(19$ to 147$)$ \\
\hline Death from CV causes & $12 \%$ & $15 \%$ & $17 \%(6$ to 28$)$ & $44(26$ to 148$)$ \\
\hline
\end{tabular}

$\ddagger C V$ = cardiovascular. Other abbreviations defined in glossary; RRR, NNT, and $\mathrm{Cl}$ calculated from data in article. 